\title{
HRJ
}

V.3 n.14 (2022)

Recebido:07/12/21

Aceito: 14/12/2021

\section{Qualidade de vida da equipe de enfermagem do centro cirúrgico no trabalho}

\author{
Mayara Gundim dos Santos ${ }^{1}$ \\ Alexandra Isabel de Amorim Lino ${ }^{2}$ \\ 1 Enfermeira, Residente de Enfermagem em Centro Cirúrgico, Escola Superior de Ciências da Saúde-ESCS, \\ Brasilia-DF, Brasil. \\ ${ }^{2}$ Enfermeira, Mestre em enfermagem, tutora da residência de Centro Cirúrgico, Hospital de Base do Distrito \\ Federal, Brasília-DF, Brasil.
}

\section{RESUMO}

Objetivo: Avaliar a qualidade de vida da equipe de enfermagem do centro cirúrgico no trabalho em um Hospital Público. Métodos: Trata-se de uma pesquisa descritiva, transversal, exploratória com abordagem, quantitativa, desenvolvida com 43 profissionais de enfermagem atuantes no centro cirúrgico de um hospital regional público do Distrito Federal, com a aplicação de um questionário sobre dados sociodemográficos e um instrumento estruturado e validado WHOQOL-Bref; a análise estatística foi realizada através da distribuição de frequência e percentual, e calculando a média por domínio. Resultados: Observa-se todos os domínios (Físico, Relações Sociais, Psicológico e Meio Ambiente/Profissional) comtemplados no questionário obtiveram baixos resultados, detectando que a qualidade de vida dos participantes em todos os aspectos analisados é abaixo dos níveis satisfatórios. Conclusão: Nota-se um impacto considerável no domínio meio ambiente e profissional, destacando que a valorização da equipe de enfermagem e a saúde desses trabalhadores ainda precisam avançar muito até alcançar níveis desejáveis.

Palavras-chave: Centro Cirúrgico; Enfermagem; Qualidade de Vida; Saúde do Trabalhador.

\section{Quality of life of the surgical center nursing staff at work}

\begin{abstract}
Objective: To assess the quality of life of the nursing staff in the operating room at work in a Public Hospital. Methods: This is a descriptive, cross-sectional, exploratory, quantitative research, developed with 43 nursing professionals working in the operating room of a public regional hospital in the Federal District, with the application of a questionnaire on sociodemographic data and a structured instrument and validated WHOQOL-Bref; statistical analysis was performed by distributing frequency and percentage, and calculating the mean per domain. Results: It is observed that all domains (Physical, Social Relations, Psychological and Environment/Professional) included in the questionnaire had low results, detecting that the quality of life of the participants in all analyzed aspects is below satisfactory levels. Conclusion: There is a considerable impact on the environment and professional domain, highlighting that the valuation of the nursing team and the health of these workers still need to go a long way to reach desirable levels.
\end{abstract}

Keywords: Surgical Center; Nursing; Quality of Life; Worker's Health. 


\section{INTRODUÇÃO}

O Centro Cirúrgico (CC) é um setor restrito e de grande complexidade dentro de uma unidade hospitalar, composto por diversas áreas, responsável por promover condições adequadas para realização de procedimentos anestésico-cirúrgicos, terapêuticos ou diagnósticos, tanto a nível eletivo quanto emergencial ${ }^{1}$.

É um cenário singular e com rotinas próprias, marcado pela execução de procedimentos invasivos e do uso de recursos materiais de alta precisão, praticabilidade e eficiência. Por ser considerado um ambiente complexo e crítico, de alta dependência da atuação individual e da equipe, assim requer profissionais capacitados e habilitados para atuar de forma eficaz, atendendo as necessidades dos pacientes ${ }^{1}$.

A equipe de enfermagem do centro cirúrgico tem como atribuições receber e atender os pacientes em todas as etapas da cirurgia, desde o pré-operatório, transoperatório e pósoperatório, com a finalidade de prestar uma assistência eficiente e de qualidade ${ }^{2}$.

O ambiente de trabalho exige técnicas assépticas rigorosas e a execução de atividades de grande responsabilidade, que se houver algum comprometimento na realização, podem ocasionar insucessos das intervenções e colocar o paciente em risco ${ }^{2}$.

O estresse das rotinas diárias, as tomadas de decisões imediatas, sobrecarga de trabalho e passar longas horas em pé durante as cirurgias, podem gerar um impacto negativo na qualidade de vida dos mesmos e na assistência prestada ao paciente ${ }^{2}$.

Sabendo ainda que no âmbito hospitalar, o CC é considerado um cenário estressante por ser fechado, frio, crítico e com alto nível de exaustão, essas condições podem levar a um desequilíbrio, físico emocional e desmotivação profissional ${ }^{2}$.

A qualidade de vida é conceito amplo, ligado ao bem-estar físico, psicológico e sociocultural dos indivíduos, que expressa saúde e longevidade. Diante disso há uma necessidade de saber gerenciar melhor os padrões impostos, o estresse, as relações interpessoais e os cuidados com a saúde, ressaltando que todo o contexto vivido diariamente 
pela equipe de enfermagem afeta diretamente a qualidade de vida no trabalho ${ }^{3}$.

Segundo Soratto et al. $(2016)^{4}$ esses profissionais precisam ser norteados sobre a importância de se autoconhecer e se reconhecer, valorizando o autocuidado para então está preparado para cuidar do outro.

Por isso, esse estudo teve como objetivo avaliar a qualidade de vida da equipe de enfermagem do centro cirúrgico.

\section{MÉTODOS}

Trata-se de uma pesquisa descritiva, transversal, exploratória com abordagem, quantitativa, realizado em um hospital público, na cidade de Brasília (DF), no qual o centro cirúrgico compreende seis salas cirúrgicas onde são realizadas, em média, oito cirurgias diárias, de caráter eletivo, de urgência e emergência.

O estudo foi constituído de 43 participantes, elencando como critérios de inclusão os profissionais de enfermagem (enfermeiros, técnicos e auxiliares de enfermagem) que apresentavam mais de 6 meses na unidade respectiva e que aceitaram participar do estudo. Foram excluídos ao todo 19 profissionais que estavam afastados do serviço por licença, atestado, férias ou que não concordaram em participar da pesquisa.

A coleta de dados foi realizada através da aplicação de dois questionários enviados por link do google forms para cada participante, sendo o primeiro elaborado pelos autores e relacionado aos dados sociodemográficos e o segundo foi o instrumento validado WHOQOLBref, para a avaliação da qualidade de vida no trabalho (modificado pela autora de 26 questões para 20 questões, sendo 6 questões para o domínio físico e saúde; 4 domínio psicológico; 3 de domínio social e pessoal; e 7 de domínio meio ambiente e profissional.

Os dados foram tabulados em planilhas do Software Excel 2010 e processados no Software SPSS (Statistical Package for the Social Sciences) versão 28.0, foi realizada uma estatística descritiva utilizando frequências e porcentis. 
Este estudo seguiu os preceitos éticos da Resolução 466/2012 do Conselho Nacional de Saúde, sendo aprovado pelo Comitê de Ética em Pesquisa da Fundação de Ensino e Pesquisa em Ciências de Saúde - FEPECS da cidade de Brasília com o CAAE 51228321.2.0000.5553.

\section{RESULTADOS}

O estudo foi realizado com profissionais de saúde de enfermagem, um total de 43 participantes que atuam em um Centro cirúrgico da região. A maioria dos participantes eram do sexo feminino $39(90,7 \%)$ e apenas $4(9,3 \%)$ do sexo masculino. Quando questionados em relação ao estado civil a maioria também eram casados 23 (54\%), seguido de 13 (30\%) solteiros, $5(12 \%)$ divorciados e apenas 1 viúvo (2\%).

Mais uma vez a maior parte dos participantes tem filhos $25(58,1 \%)$, enquanto 18 $(41,9 \%)$ informaram não ter filhos. Quanto a formação $28(65,1 \%)$ informaram ter ensino superior, 6 (14\%) ensino superior incompleto e 9 (20,9\%) ensino médio.

No que se refere a renda percapita, 27 (62,7\%) recebem mais de 4 salários mínimos, $14(32,6 \%)$ de 2 a 4 salários mínimos e $2(4,7 \%)$ de 1 a 2 salários mínimos.

Tabela 1: Apresenta as variáveis relaccionadas aos dados sociodemográficos, Brasília, Brasil, 2021.

\begin{tabular}{llcc}
\multicolumn{1}{c}{ Variáveis sóciodemográficas } & N & \% \\
\hline \multirow{2}{*}{ Sexo } & Feminino & 39 & 90,7 \\
& Masculino & 4 & 9,3 \\
& Total & 43 & 100,0 \\
\cline { 2 - 4 } Estado civil & Casado & 23 & 54,0 \\
& Solteiro & 13 & 30,0 \\
& Viúvo & 1 & 2,0 \\
Tem filhos & Divorciado & 5 & 12,0 \\
& Não avaliado & 1 & 2,0 \\
& Total & 43 & 100,0 \\
\cline { 2 - 4 } & Não & 18 & 41,9 \\
& Sim & 25 & 58,1 \\
& Total & 43 & 100,0 \\
\cline { 2 - 4 } & Ensino superior & 28 & 65,1 \\
& Ensino superior incompleto & 6 & 14,0 \\
& Ensino médio & 9 & 20,9 \\
& Total & 43 & 100,0 \\
\cline { 2 - 4 } & & &
\end{tabular}




$\begin{array}{llcc} & \text { Mais de } 4 \text { salários mínimos } & 27 & 62,7 \\ \text { Renda percapita } 2 \text { a } 3 \text { salários mínimos } & 14 & 32,6 \\ & \text { De 1 a 2 salários mínimos } & 2 & 4,7 \\ & \text { Total } & 43 & 100,0\end{array}$

Fonte: Dados da pesquisa realizada pela autora, a partir do questionário sobre perfil sociodemográfico.

Na tabela 2, observamos as variáveis relacionadas com o trabalho, em relação à categoria profissional, a maioria $31(72,1 \%)$ é técnica de enfermagem e $12(27,9 \%)$ enfermeiros. No que se refere ao turno de trabalho $33(76,7 \%)$ trabalham durante o dia e 10 $(23,3 \%)$ no turno noturno.

A locomoção para o trabalho pode ser cansativa, porém apenas 9 (20,9\%) moram em cidade diferente do trabalho, sendo que $33(79,1 \%)$ residem na mesma cidade onde trabalham.

Em relação a possuírem outro vínculo, 11 (25,6\%) relataram ter outro vínculo e 32 $(74,4 \%)$ não possuem outro vínculo. Porém esse número aumenta quando questionados em relação a realização de horas extra, 26 (60,5\%) fazem horas extra, enquanto 17 (39,5\%) informaram não realizar extra.

Na avaliação quanto ao nível de satisfação (compreendendo as notas de 0 a 10) no seu trabalho, a maioria apontou notas acima de 7, sendo que 9 (20,9\%) atribuíram nota 7, 14 $(32,6 \%)$ nota $8,8(18,6 \%)$ nota $9,1(2,3 \%)$ nota 9,5 e $6(14 \%)$ nota 10.

Tabela 2: Apresenta as variáveis relacionadas ao trabalho, Brasília, Brasil, 2021.

\begin{tabular}{|c|c|c|c|}
\hline \multicolumn{2}{|c|}{ Variáveis relacionadas ao trabalho } & \multirow{2}{*}{$\frac{\mathrm{N}}{12}$} & \multirow{2}{*}{$\frac{\%}{27,9}$} \\
\hline \multirow{3}{*}{ Categoria profissional } & Enfermeiro(a) & & \\
\hline & $\begin{array}{l}\text { Téc. (a) de } \\
\text { enfermagem }\end{array}$ & 31 & 72,1 \\
\hline & Total & 43 & 100,0 \\
\hline \multirow{3}{*}{ Turno de trabalho } & Diurno & 33 & 76,7 \\
\hline & Noturno & 10 & 23,3 \\
\hline & Total & 43 & 100,0 \\
\hline \multirow{3}{*}{ Trabalha na cidade onde mora } & Sim & 34 & 79,1 \\
\hline & Não & 9 & 20,9 \\
\hline & Total & 43 & 100,0 \\
\hline \multirow{3}{*}{$\begin{array}{l}\text { Possui outro vínculo } \\
\text { empregatício }\end{array}$} & Sim & 11 & 25,6 \\
\hline & Não & 32 & 74,4 \\
\hline & Total & 43 & 100,0 \\
\hline \multirow{2}{*}{ Faz hora extra } & Sim & 26 & 60,5 \\
\hline & Não & 17 & 39,5 \\
\hline
\end{tabular}




\begin{tabular}{lcc} 
Total & 43 & 100,0 \\
\hline 3,00 & 1 & 2,3 \\
5,00 & 1 & 2,3 \\
6,00 & 3 & 7,0 \\
7,00 & 9 & 20,9 \\
8,00 & 14 & 32,6 \\
9,00 & 8 & 18,6 \\
9,50 & 1 & 2,3 \\
10,00 & 6 & 14,0 \\
Total & 43 & 100,0
\end{tabular}

Fonte: Fonte: Dados da pesquisa realizada pela autora, a partir do questionário sobre variáveis relacionadas ao trabalho.

As tabelas 3, 4 e 5 trazem as respostas às sentenças da qualidade de vida, constando as frequências e porcentis avaliado pelo instrumento WHOQOL-BREF, através dos domínios físico, psicológico, relações sociais/pessoais e meio ambiente/profissional.

Nesta é possível observar que a maioria dos participantes avaliam como boa 25 $(58,1 \%)$ e muito boa $11(25,6 \%)$ a qualidade de vida, assim como a satisfação em relação ao domínio físico/saúde $8(18,6 \%)$ muito satisfeito e $24(55,8 \%)$ satisfeito, com respostas positivas quanto a dor física $(32,6 \%)$ ausente, e necessidade de tratamento na vida diária $(34,9 \%)$ sem necessidade. Quanto a satisfação com o sono $22(51,1 \%)$ dizem estar satisfeito, porém quando questionado sobre a energia suficiente para o dia a dia percebe-se um intermédio em 19 (44,2\%) das repostas, assim ainda 30 (69,8\%) diz está satisfeito com sua capacidade de desempenhar atividades do trabalho, enquanto 9 (20,9\%) nem satisfeito e nem insatisfeito.

Tabela 3: Apresenta as variáveis relacionadas à qualidade de vida, Brasília, Brasil, 2021.

\begin{tabular}{llcc}
\hline \multicolumn{1}{c}{ Variáveis relacionadas à qualidade de vida (1/3) } & $\mathbf{N}$ & \% \\
\hline & Muito boa & 11 & 25,6 \\
Como você avalia sua qualidade de vida & Boa & 25 & 58,1 \\
& Nem ruim nem boa & 4 & 9,3 \\
Está satisfeito com sua saúde & Ruim & 3 & 7,0 \\
& Total & 43 & 100,0 \\
\cline { 2 - 4 } & Muito satisfeito & 8 & 18,6 \\
& Satisfeito & 24 & 55,8 \\
& Nem satisfeito nem & 7 & 16,3
\end{tabular}




\begin{tabular}{|c|c|c|c|}
\hline \multirow{6}{*}{ Tem dor física ela impede você de fazer o que precisa } & Total & 43 & 100,0 \\
\hline & Mais ou menos & 12 & 27,9 \\
\hline & Nada & 14 & 32,6 \\
\hline & Muito pouco & 12 & 27,9 \\
\hline & Bastante & 5 & 11,6 \\
\hline & Total & 43 & 100,0 \\
\hline \multirow{6}{*}{ Você faz tratamento para levar sua vida diária } & Mais ou menos & 8 & 18,6 \\
\hline & Nada & 15 & 34,9 \\
\hline & Muito pouco & 14 & 32,6 \\
\hline & Bastante & 5 & 11,6 \\
\hline & Extremamente & 1 & 2,3 \\
\hline & Total & 43 & 100,0 \\
\hline \multirow{6}{*}{ Quão satisfeito você está com seu sono } & Satisfeito & 22 & 51,1 \\
\hline & Insatisfeito & 8 & 18,6 \\
\hline & Muito insatisfeito & 1 & 2,3 \\
\hline & $\begin{array}{l}\text { Nem satisfeito nem } \\
\text { insatisfeito }\end{array}$ & 10 & 23,3 \\
\hline & Muito satisfeito & 2 & 4,7 \\
\hline & Total & 43 & 100,0 \\
\hline \multirow{7}{*}{ Você tem energia suficiente para seu dia a dia } & Muito & 16 & 37,2 \\
\hline & Médio & 19 & 44,2 \\
\hline & Muito pouco & 4 & 9,3 \\
\hline & Nada & 1 & 2,3 \\
\hline & Completamente & 3 & 7,0 \\
\hline & Total & 43 & 100,0 \\
\hline & Satisfeito & 30 & 69,8 \\
\hline \multirow{5}{*}{$\begin{array}{l}\text { Quão satisfeito com capacidade de desempenhar suas } \\
\text { atividades do trabalho }\end{array}$} & Insatisfeito & 1 & 2,3 \\
\hline & Muito insatisfeito & 1 & 2,3 \\
\hline & $\begin{array}{l}\text { Nem satisfeito nem } \\
\text { insatisfeito }\end{array}$ & 9 & 20,9 \\
\hline & Muito satisfeito & 2 & 4,7 \\
\hline & Total & 43 & 100,0 \\
\hline
\end{tabular}

Fonte: Dados da pesquisa realizada pela autora, a partir do questionário de qualidade de vida - WHOQOL: World Health Organization Quality of Life Assessment.

A tabela 4 apresenta respostas relacionadas ao domínio psicológico e relações pessoais e sociais, nota- se que no domínio psicológico, 20 (46,5\%) afirmam que conseguem tem bastante facilidade em se concentrar e $18(41,9)$ relatam ter um pouco de dificuldade. Sobre aceitar a aparência física 15 (34,9\%) dizem que completamente e 12 (27,9\%) respondem que muito, porém $11(25,6 \%)$ não aceitam a sua aparência e relatam que por esse motivo tem a autoestima afetada. Observa-se que questionados sobre ter sentimentos negativos com frequência $32(74,5 \%)$ responderam que algumas vezes e $5(11,6 \%)$ frequentemente, o que pode ser um fator negativo durante a assistência prestada aos pacientes, $(44,2 \%)$ dizem que aproveita a vida bastante e $(37,2 \%)$ mais ou menos. Entretanto no que se 
refere ao domínio pessoal e social, $28(65,1 \%)$ garantem que estão satisfeitos consigo mesmo no trabalho e $21(48,8 \%)$ demonstram satisfação com apoio em que recebe dos seus colegas de trabalho ao mesmo tempo em que $24(55,8 \%)$ dizem também está contente com suas relações pessoais.

Tabela 4: Apresenta a continuidade das variáveis relacionadas à qualidade de vida, Brasília, Brasil, 2021.

\begin{tabular}{|c|c|c|c|}
\hline (cont.2/3) Variáveis relacionadas à qualidade de vida & & $\mathbf{N}$ & $\%$ \\
\hline \multirow{5}{*}{ Quanto você se consegue se concentrar } & Mais ou menos & 18 & 41,9 \\
\hline & Muito pouco & 4 & 9,3 \\
\hline & Bastante & 20 & 46,5 \\
\hline & Extremamente & 1 & 2,3 \\
\hline & Total & 43 & 100,0 \\
\hline \multirow{7}{*}{ Você aceita sua aparência física } & Completamente & 15 & 34,9 \\
\hline & Muito & 12 & 27,9 \\
\hline & Médio & 4 & 9,3 \\
\hline & Muito pouco & 1 & 2,3 \\
\hline & Nada & 11 & 25,6 \\
\hline & Total & 43 & 100,0 \\
\hline & Algumas vezes & 32 & 74,5 \\
\hline \multirow{5}{*}{ Tem sentimentos negativos com frequência } & Nunca & 4 & 9,3 \\
\hline & Frequentemente & 5 & 11,6 \\
\hline & Sempre & 1 & 2,3 \\
\hline & $\begin{array}{l}\text { Muito } \\
\text { frequentemente }\end{array}$ & 1 & 2,3 \\
\hline & Total & 43 & 100,0 \\
\hline \multirow{4}{*}{ Quanto você aproveita a vida } & Mais ou menos & 16 & 37,2 \\
\hline & Muito pouco & 7 & 16,3 \\
\hline & Bastante & 19 & 44,2 \\
\hline & Extremamente & $\begin{array}{c}1 \\
43\end{array}$ & $\begin{array}{c}2,3 \\
1000\end{array}$ \\
\hline \multirow{4}{*}{ Quão satisfeito está com apoio dos seus colegas de trabalho } & Satisfeito & 21 & 48,8 \\
\hline & $\begin{array}{l}\text { Nem satisfeito } \\
\text { nem insatisfeito }\end{array}$ & 15 & 34,9 \\
\hline & Muito satisfeito & 7 & 16,3 \\
\hline & Total & 43 & 100,0 \\
\hline \multirow{7}{*}{ Quão satisfeito está consigo mesmo no trabalho } & Satisfeito & 28 & 65,2 \\
\hline & Insatisfeito & 1 & 2,3 \\
\hline & $\begin{array}{l}\text { Nem satisfeito } \\
\text { nem insatisfeito }\end{array}$ & 9 & 20,9 \\
\hline & Muito satisfeito & 5 & 11,6 \\
\hline & Total & 43 & 100,0 \\
\hline & Satisfeito & 24 & 55,8 \\
\hline & $\begin{array}{l}\text { Nem satisfeito } \\
\text { nem insatisfeito }\end{array}$ & 13 & 30,2 \\
\hline \multirow{2}{*}{ Quão satisfeito está com suas relações pessoais } & Muito satisfeito & 6 & 14,0 \\
\hline & Total & 43 & 100,0 \\
\hline
\end{tabular}

Fonte: Dados da pesquisa realizada pela autora, a partir do questionário de qualidade de vida - WHOQOL: World Health Organization Quality of Life Assessment. 
A tabela 5 estão descritas respostas relativas aos domínios relações pessoais/sociais e meio ambiente e profissional, verifica-se que $35(81,3 \%)$ declara está satisfeito com o seu trabalho, simultaneamente em que $27(62,8 \%)$ demonstram uma satisfação intermediária quando questionados sobre o quão saudável é o ambiente de trabalho. $24(55,8 \%)$ dos participantes alegam ter uma base financeira sólida suficiente para satisfazer as suas necessidades, $(62,8 \%)$ afirmam bastante segurança na realização de atividades relacionadas ao trabalho, sobre a realização de atividades de lazer nota-se um resultado positivo em que 22 $(51,2 \%)$ falam que muito e $11(25,6)$ completamente. Percebe-se que na última pergunta eles foram questionados sobre ter uma qualidade de vida afetada pelo trabalho, 23 (53,5\%) diz que algumas vezes e 9 (20,9\%) não expressam muita satisfação ao responderem que frequentemente tem a qualidade de vida afetada negativamente pelo trabalho.

Tabela 5: Apresenta a continuidade das variáveis relacionadas à qualidade de vida, Brasília, Brasil, 2021.

\begin{tabular}{|c|c|c|c|}
\hline \multicolumn{2}{|l|}{ (cont. 3/3) Variáveis relacionadas à qualidade de vida } & $\mathbf{N}$ & $\%$ \\
\hline \multirow{5}{*}{ Quão satisfeito você está com o seu trabalho } & Satisfeito & 35 & 81,3 \\
\hline & Insatisfeito & 2 & 4,7 \\
\hline & $\begin{array}{l}\text { Nem satisfeito } \\
\text { em insatisfeito }\end{array}$ & 2 & 4,7 \\
\hline & Muito satisfeito & 4 & 9,3 \\
\hline & Total & 43 & 100,0 \\
\hline \multirow{5}{*}{ Quão saudável é seu ambiente de trabalho } & Mais ou menos & 27 & 62,8 \\
\hline & Nada & 2 & 4,7 \\
\hline & Muito pouco & 5 & 11,6 \\
\hline & Bastante & 9 & 20,9 \\
\hline & Total & 43 & 100,0 \\
\hline \multirow{5}{*}{ Você tem dinheiro suficiente para satisfazer suas necessidades } & Completamente & 11 & 25,6 \\
\hline & Muito & 24 & 55,8 \\
\hline & Médio & 6 & 14,0 \\
\hline & Muito pouco & 2 & 4,6 \\
\hline & Total & 43 & 100,0 \\
\hline \multirow{5}{*}{$\begin{array}{l}\text { Se sente seguro na realização das atividades diárias relacionadas ao } \\
\text { trabalho }\end{array}$} & Mais ou menos & 11 & 25,6 \\
\hline & Muito pouco & 1 & 2,3 \\
\hline & Bastante & 27 & 62,8 \\
\hline & Extremamente & 4 & 9,3 \\
\hline & Total & 43 & 100,0 \\
\hline \multirow{4}{*}{ Você realiza atividades de lazer } & Completamente & 11 & 25,6 \\
\hline & Muito & 22 & 51,2 \\
\hline & Médio & 9 & 20,9 \\
\hline & Muito pouco & 1 & 2,3 \\
\hline
\end{tabular}




\begin{tabular}{llcc}
\hline & & & 53,5 \\
Seu trabalho afeta sua qualidade de vida & Algumas vezes & 23 & 18,6 \\
& Nunca & 8 & 20,9 \\
& Frequentemente & 9 & 7,0 \\
& Sempre & 3 & 43 \\
\hline
\end{tabular}

Fonte: Dados da pesquisa realizada pela autora, a partir do questionário de qualidade de vida - WHOQOL: World Health Organization Quality of Life Assessment.

Para avaliação da qualidade de vida foi realizado também o cálculo da média por domínio de acordo com o instrumento WHOQOL-BREF, o domínio - físico/saúde obteve uma média de $(3,06)$, psicológico $(3,21)$, relações pessoais/sociais $(3,81)$ e meio ambiente e profissional $(2,99)$. Esses resultados foram avaliados com base na classificação final do questionário onde, necessita melhorar (quando for 1 até 2,9); regular (3 até 3,9); boa (4 até 4,9) e muito boa (5).

\section{DISCUSSÃO}

Há muito tempo o tema qualidade de vida $(\mathrm{QV})$ vem ganhando espaço no cenário científico, porém quando direcionada aos trabalhadores de enfermagem, constata-se um declínio de investigações. Esses profissionais tem sua QV avaliada em alguns estudos com diferentes pontos de vista, porém a maioria com foco nas condições de trabalho em turno, condições de vida, saúde física e psicológica ${ }^{5}$.

Essas pesquisas têm sido realizadas com diferentes grupos e em vários aspectos, ratificando a importância de verificar quais os fatores que interferem nos resultados alcançados ${ }^{6}$.

Nesse estudo $(90,7 \%)$ foi prevalente o sexo feminino e isso se deve ao contexto histórico da enfermagem. Uma pesquisa recente garante que esse panorama vem se transformando, acerca do aumento do número de homens nos cursos superior e técnico de enfermagem ${ }^{3}$. 
O fato de a maioria dos profissionais pesquisados ser (54\%) casado e a maior parte $(58,1 \%)$ possuírem filhos, mostra que esses elementos podem interferir tanto positivamente quanto negativamente na qualidade de vida delas. Segundo Stumm et al. $2013 \mathrm{em}$ pesquisas do dia-a-dia de mulheres-mães-trabalhadoras de enfermagem, identificaram interferência da profissão na vida delas, além de analisarem a perceção dessa realidade, se considerado o tipo de atividade que desenvolvem, e que frequentemente, desencadeiam mudanças importantes na rotina familiar ${ }^{7}$

Quanto à formação dos profissionais, sobrelevar $(65,1 \%)$ os com o ensino superior completo, resultado que evidencia investimento na formação profissional, em busca de maiores índices de satisfação, melhores salários e condições de vida, com resultados positivos na qualidade de vida.

Observou-se que a maior parte dos profissionais participantes $(62,7 \%)$ recebe a renda percapita mais de 4 salários-mínimos. Esses dados eram esperados, visto que a maioria dos entrevistados exerce a função de enfermeiro.

Em relação à categoria profissional a dominância $(72,1 \%)$ de técnicos de enfermagem se justifica pelo motivo de compor o maior número de profissionais da equipe.

No que se refere ao turno de trabalho, prevaleceu $(76,7 \%)$ os profissionais que trabalham durante o dia. Simões 2013 aponta que isso ocorre porque grande parte dos profissionais que trabalham à noite e dormem no decorrer do dia, criam situações em que seu horário de trabalho entra em divergência com os horários estabelecidos pela sociedade e pelo seu próprio organismo, acarretando certos prejuízos à saúde ${ }^{8}$.

O domínio $(79,1 \%)$ de participantes que residem na mesma cidade onde trabalham, é enxergado como ponto positivo. Já que de acordo com Soratto et al., 2016 essa pequena porcentagem que afirmam morar em cidade diferente do trabalho, tem maior risco de estresse, cansaço e insatisfação com o serviço, que pode resultar em absenteísmo ${ }^{4}$. 
No que tange possuir ou não outro vínculo, o predomínio $(74,4 \%)$ estar nos profissionais que garantem não possuir outro vinculo. Enquanto, o número de participantes $(60,5 \%)$ que realizam horas extras se destaca com a maior porcentagem.

Conforme Soares, Oliveira e Sousa, 2017 em razão dos baixos salários, grande parte dos profissionais da enfermagem é obrigada a realizar horas extras, o que leva esses profissionais a permanecerem no ambiente dos serviços de saúde a maior parte do tempo de suas vidas produtivas. Essa situação leva ao aumento do período de exposição aos riscos existentes nesses locais, sendo capaz de provocar prejuízo para sua qualidade de vida ${ }^{6}$.

$\mathrm{Na}$ avaliação quanto ao nível de satisfação com o trabalho, o fato das notas de 0 a 10 a nota maior dos profissionais estar em $(32,6 \%)$ nota 8 , é um bom resultado que, certamente, se consiste em avaliação positiva para qualidade de vida da equipe de enfermagem. Para Vieira (2017), uma condição que influencia a satisfação com o trabalho, é a remuneração financeira digna e compatível com as atividades exercidas no $\mathrm{CC}$, além de responder às necessidades básicas, concede um papel de reconhecimento pelas ações desenvolvidas ${ }^{9}$.

Quanto a avaliação da qualidade de vida dos profissionais pesquisados, constata-se que $58,1 \%$ avaliam como boa e $25,6 \%$ como muito boa, o que é positivo tanto para eles, quanto para instituição de saúde, especificamente para o centro cirúrgico.

De modo geral, quando calculado a média por domínio notou-se que a qualidade de vida dos participantes está abaixo dos níveis satisfatórios.

O domínio físico (tabela 3) apresentou uma média final de 3,06, classificada como regular na avaliação final do questionário WHOQOL-BREF, a maioria dos profissionais afirmam satisfação com a sua saúde, 18,6\% muito satisfeito e 55,8\% satisfeito, o que é um resultado positivo, já que de acordo com um estudo epidemiológico realizado no Rio Grande do Sul o principal fator que determina uma qualidade de vida ruim é a perceção de não ter saúde, pois pode afetar o domínio Físico ${ }^{10}$. 
Percebe-se que em relação a dor física e necessidade de tratamento para levar a vida diária teve-se bons resultados. Onde (32,6\%) afirmam dor ausente, $(27,9 \%)$ Muito pouco, e sobre a necessidade de tratamento na vida diária, $(34,9 \%)$ Nada e (32,6\%) Muito pouco.

Ao mesmo tempo em que $51,1 \%$ demonstram uma satisfação com o sono, $44,2 \%$ alegam uma satisfação intermediaria quanto a ter energia suficiente para o dia-dia.

Algumas pesquisas relatam que a fadiga afeta indivíduos de todas as faixas etárias, que enfrentam jornadas prolongadas de trabalho, com turnos não favoráveis, e requerem bastante esforço físico e mental. Descreve também que a presença de dor, dependência de medicação, insatisfação com o sono, incapacidade para o trabalho e falta de energia para realizar atividades diárias, são condições contribuintes para a falta de qualidade de vida destes profissionais, que acabam recorrendo com frequência a tratamentos medicamentosos, muitas vezes utilizando-se da automedicação, e que todos esses fatores acabam respingando de forma negativa na assistência prestada ao paciente ${ }^{5}$.

Portanto, deve se levar em consideração a perceção que os profissionais tem da sua saúde, pois elas influenciam diretamente para uma melhor qualidade de vida ${ }^{10}$.

O domínio psicológico (tabela 4) obteve média de 3,21, classificada como "Regular", ele aborda todos os aspectos relacionados a compreende o aprender, a memória e concentração, sentimentos, à satisfação pessoal e motivação no trabalho.

Os resultados obtidos nesse domínio expõem a forma como os profissionais avaliaram as variáveis. Se concentram (46,5\% bastante e 41,9\% mais ou menos), aceitam sua aparência física $(34,9 \%$ completamente e $27,9 \%$ muito), tem sentimentos negativos $(74,5 \%$ algumas vezes e $11,6 \%$ frequentemente), quanto a aproveitar a vida 44,2\% responderam "bastante e $37,2 \%$ "mais ou menos".

Identificou-se um quesito merecedor de atenção e de ações que é o referente a sentimentos negativos, quando questionados sobre ter sentimentos negativos mais da metade 
dos participantes $74,5 \%$ responderam algumas vezes.

Um estudo aponta que os aspectos emocionais, vitalidade e saúde mental são comprometidos, devido as características organizacionais e de rotinas do centro cirúrgico, como a cobrança por produtividade e agilidade, para maior rotatividade de cirurgias, longas horas auxiliando procedimentos cirúrgicos, a falta de reconhecimento e desvalorização profissional, que acabam favorecendo sentimentos negativos, tais como ansiedade, tensão e irritabilidade ${ }^{11}$.

O Domínio de Relações Pessoais/Sociais (Tabela 4) foi calculado uma média de 3,81, sendo caracterizada pela classificação de avaliação do questionário como "Regular", ele refere-se ao nível de satisfação dos pesquisados com pessoas do círculo social, com as relações pessoais e satisfação consigo mesmo no trabalho, nesse sentido, mais de 50\% responderam satisfeitos. Enquanto ao apoio dos colegas de trabalho 48,8\% dizem está satisfeitos e 34,9\% nem satisfeitos e nem insatisfeitos.

O apoio dos colegas é um fator que contribuí para o desenvolvimento do trabalho, a comunicação é essencial, a enfermagem em si exige boas relações com toda a equipe.

Madrid e Glanzner (2021) destacam que a falta de compromisso e pontualidade de alguns profissionais do centro cirúrgico, pode acabar sobrecarregando os colegas de trabalho, causando conflitos entre a equipe e insatisfação com as relações ${ }^{11}$.

O domínio meio ambiente e profissional apresentou uma média de 2,99, classificada como "Necessita Melhorar" de acordo com a classificação final do questionário.

A análise das variáveis do domínio Meio Ambiente e Profissional mostrou que os profissionais do $\mathrm{CC}$ do respectivo hospital responderam estar "satisfeitos" com o trabalho (81,3\%), enquanto $(62,8 \%)$ "mais ou menos" satisfeitos em relação ao ambiente de trabalho. Alguns artigos ainda enfatizam também o ambiente de trabalho como fator a ser analisado já que é considerado importante para satisfação e bem-estar do trabalhador, e quando não 
considerado saudável pode afetar a qualidade de vida ${ }^{5}$.

Questionados sobre ter dinheiro para satisfazer suas necessidades, e realizar atividades de lazer, avaliaram as variáveis referentes ao respectivo domínio, na intensidade "muito" e "completamente", dinheiro suficiente para se satisfazer $(55,8 \%$ e $25,6 \%)$, lazer (51,2\% e 25,6\%). Já sobre se sentir seguro 62,8\% responderam que bastante.

De acordo com Vitorino (2014) foi avaliado em 2011 a satisfação profissional em um hospital no Japão, e demonstrou que um dos fatores que colaboraram positivamente com o bem-estar profissional de enfermagem foi renda. Isso justifica as respostas positivas nas variáveis citadas anteriormente, já que mais de 50\% dos participantes desse estudo afirmam ter uma renda com mais de quatro salários mínimos ${ }^{10}$.

Sobre ter uma qualidade de vida afetada pelo trabalho, 53,5\% responderam "algumas vezes e $20,9 \%$ "frequentemente". Confirmando esse resultado, um estudo em um hospital escola da cidade de São Paulo para avaliar a qualidade de vida dos trabalhadores de enfermagem, mostrou que $80 \%$ dos profissionais não estão satisfeitos com a sua qualidade de vida, apontando que esse aspecto está longe de ser modificado e alcançar um nível desejável ${ }^{7}$. O maior impacto no domínio meio ambiente e profissional pode ser justificado pela satisfação intermediaria com o ambiente de trabalho, seguido pela insatisfação sobre ter uma qualidade de vida afetada pelo trabalho.

A equipe de enfermagem do centro cirúrgico desempenham suas atribuições nesse ambiente restrito, intenso, frio e rotineiro, com sua competência e responsabilidade ligada a exposição de agentes estressores, ausência de equipamentos para o trabalho, déficit de profissionais qualificados, demanda alta de cirurgias, estado fisiológico e mental dos pacientes. Dessa forma, profissionais expostos a essas condições tem uma repercussão negativa na qualidade de vida no trabalho, muitas vezes refletindo na assistência prestada ${ }^{12}$.

Ressaltando que esse resultado igualmente salienta os vários aspectos envolvidos na 
QV, ou seja, a subjetividade e a multidimensionalidade que a mesma abrange e percebe que todos os domínios de acordo com a classificação de resultado final do questionário precisam obter resultados mais satisfatórios.

\section{CONCLUSÃO}

Os dados obtidos nessa pesquisa demonstram que os profissionais de saúde que atuam no centro cirúrgico, avaliaram sua qualidade de vida no trabalho como boa, porém na análise das variáveis que compreende cada domínio, comprovam que todas elas podem ser melhoradas e alcançar níveis mais satisfatórios, principalmente o domínio meio ambiente e profissional.

A qualidade de vida do profissional de enfermagem ainda é um desafio, faz se necessária, à implementação de programas e políticas institucionais que contribuam para à melhoria da mesma e que os fatores agravantes sejam minimizados. Uma melhor qualidade de vida dos trabalhadores de enfermagem pode contribuir para a instituição, visto que indivíduos satisfeitos tem um bom desenvolvimento e produtividade, resultando, assim, em qualidade da assistência por ele prestada.

Sugere-se que sejam realizadas novas pesquisas sobre a temática, para que haja mais informações a respeito, no intuito de diagnosticar problemas e determinar soluções que otimizem a qualidade de vida desses profissionais.

\section{REFERÊNCIAS}

1- Martins FZ, Dall'Agnol CM. Surgical center: challenges and strategies for nurses in managerial activities. Rev Gaúcha Enferm. 2016;37(4):e56945. Available from: https://doi.org/10.1590/1983-1447.2016.04.56945.

2- Miranda SMM. O nível de estresse do profissional de enfermagem que atua no centro 
cirúrgico em um hospital privado do Distrito Federal. repositoriouniceubbr [Internet]. 2017 [cited 2021 Dec 5]; Disponível em: https://repositorio.uniceub.br/jspui/handle/235/11750.

3- Carvalho A de MB, Cardoso JA, Silva FAA da, Lira JAC, Carvalho SM. Qualidade de vida no trabalho da equipe de enfermagem do centro cirúrgico. Enfermagem em Foco [Internet]. 2018 Nov 26 [cited 2021 Dec 5];9(3). Disponível em: http://revista.cofen.gov.br/index.php/enfermagem/article/view/1159.

4- Soratto MT, Souza MP, Mattos SB, Ceretta LB, Gomes KM, Correa SM. o estresse da equipe de enfermagem no centro cirúrgico. Revista Interdisciplinar de Estudos em Saúde [Internet]. 2016 Jul 4 [cited 2021 Dec 5];5(1):179-92. Disponível em: https://periodicosuniarp.com.br/ries/article/view/717.

5- Silva AE, Lima PKM, Oliveira C. Avaliação da qualidade de vida dos profissionais de enfermagem nível médio em unidade de terapia intensiva. Revista de Enfermagem do CentroOeste Mineiro. 2016 Dec 2;6(3). Available from: DOI:10.19175/recom.v6i3.1000.

6- Soares LMP, Oliveira VC, Souza LAA. Qualidade de vida dos profissionais atuantes no centro cirúrgico em Patos de Minas - MG. Revista Acta Científica. 2018;9, [cited 2021 Dec 5]. Available from: https://doi.galoa.com.br/sites/default/files/ac9/11.pdf.

7- Stumm EMF, Nogueira G de M, Kirchner RM, Guido L de A, Ubessi LD. Qualidade de vida de profissionais em um centro cirúrgico. Enfermería Global [Internet]. 2013 Mar 25 [cited 2021 Dec 5];12(2). Available from: https://revistas.um.es/eglobal/article/view/eglobal.12.2.14198.

8- Rita de CMS. Qualidade de vida dos profissionais de enfermagem do período noturno: uma revisão integrativa. Pucgoiasedubr [Internet]. 2013 [cited 2021 Dec 5]; Available from: http://tede2.pucgoias.edu.br:8080/handle/tede/2981.

9- Giovana CV. O papel da satisfação profissional na qualidade de vida do enfermeiro. UNIRIO centro de ciências biológicas e da saúde -ccbs, 2017 dez. [Internet]. [cited 2021 Dec 5]. 
http://www.repositoriobc.unirio.br:8080/xmlui/bitstream/handle/unirio/11441/DISSERTA\%c 3\%87\%c3\%830\%20GIOVANAvers\%c3\%a30\%20final.pdf?sequence=1\&isAllowed=y.

10- Vitorino LM, Monteiro FP, Silva JV, Dias EN, Santos AEO. Qualidade de vida da equipe de enfermagem em unidades de urgência e emergência. Revista de Ciências Médicas. 2014 Oct 24;23(2):83. Available from: http://dx.doi.org/10.24220/2318-0897v23n2a2527.

11- Madrid BP, Glanzner CHO. trabalho da equipe de enfermagem no centro cirúrgico e os danos relacionados à saúde. Rev Gaúcha Enferm. 2020;42(esp):e20200087. Available from: https://doi.org/10.1590/1983-1447.2020.20200087.

12-Lucena MS, De Lima TNB, De Souza JLM, Carvalho ME de L, Taurino IJM, Ferreira DRA, et al. Qualidade de vida no trabalho do enfermeiro atuante no bloco cirúrgico. Revista Eletrônica Acervo Saúde. 2020 Apr 23;(47):e2303. Available from: https://doi.org/10.25248/reas.e2303.2020. 